\title{
Single-photon-level light storage in cold atoms using the Autler-Townes splitting protocol
}

\author{
Erhan Saglamyurek, ${ }^{1, *}$ Taras Hrushevskyi, ${ }^{1}$ Logan Cooke, ${ }^{1}$ Anindya Rastogi, ${ }^{1}$ and Lindsay J. LeBlanc ${ }^{1,2, \dagger}$ \\ ${ }^{1}$ Department of Physics, University of Alberta, Edmonton, Alberta, Canada T6G $2 E 1$ \\ ${ }^{2}$ Canadian Institute for Advanced Research, Toronto, Ontario, Canada
}

(Received 14 May 2019; published 11 September 2019)

\begin{abstract}
Broadband spin-photon interfaces for the long-lived storage of photonic quantum states are key elements for quantum information technologies. Yet, the reliable operation of such memories in the quantum regime is challenging due to photonic noise arising from technical and/or fundamental limitations in the storage-and-recall processes controlled by strong electromagnetic fields. Here, we experimentally implement a single-photon-level spin-wave memory in a laser-cooled rubidium gas, based on the recently proposed Autler-Townes splitting (ATS) protocol. We demonstrate the storage of 20-ns-long laser pulses, each containing an average of 0.1 photons, for $200 \mathrm{~ns}$ with an efficiency of $12.5 \%$ and a signal-to-noise ratio above 30 . Notably, the robustness of ATS spin-wave memory against motional dephasing allows for an all-spatial filtering of the control-field noise, yielding an ultralow unconditional noise probability of $3.3 \times 10^{-4}$, without the complexity of spectral filtering. These results highlight that broadband ATS memory in ultracold atoms is a preeminent option for storing quantum light.
\end{abstract}

DOI: 10.1103/PhysRevResearch.1.022004

Large-scale quantum networks require quantum memories for storage and on-demand recall of photonic quantum states at timescales over milliseconds. Interfacing nonclassical light with these memories is necessary, but has proven to be difficult for two reasons: the substantial mismatch between the inherently large bandwidth of quantum light (from most popular single-photon sources) and the narrow acceptance bandwidth of well-studied atomic memories, and the unfaithful storage and recall processes due to photonic noise introduced by memory itself, which may degrade or fully destroy the quantum nature of the stored light. This noise is particularly problematic with on-demand memories that require control electromagnetic fields, and is typically much more detrimental for broadband implementations [1-3].

A promising approach to noise-free broadband memory is a family of photon-echo-based protocols that feature inherently fast (nonadiabatic) memory operation [4-6]. The controlled reversible inhomogeneous broadening (CRIB) [7,8] and gradient echo memory (GEM) $[9,10]$ are widely studied protocols that rely upon the absorption of light via artificially broadened spectral features controlled by external electric or magnetic field gradients. However, implementing a broadband CRIB or GEM memory is technically challenging due to the infeasibility of large field gradients with rapid switching times. Although a variant of GEM [11,12] circumvents this issue by using controlled ac Stark shifts instead of electric or magnetic field gradients, it does not offer the inherent high-

\footnotetext{
*saglamyu@ualberta.ca

†lindsay.leblanc@ualberta.ca
}

Published by the American Physical Society under the terms of the Creative Commons Attribution 4.0 International license. Further distribution of this work must maintain attribution to the author(s) and the published article's title, journal citation, and DOI. speed operation of the original photon-echo schemes. Another viable solution lies in the atomic frequency comb (AFC) technique, as this approach relies on tailoring a comb-shaped spectral feature for light absorption without needing controlled broadening [13]. To this end, broadband AFC quantum memories have been successfully demonstrated for the highfidelity storage of entangled photons in the GHz-bandwidth regime using an ensemble of two-level rare-earth (RE) ions in solids [14-17]. But, intrinsically short and preprogrammed storage times in these memories restrict their use to specific applications $[18,19]$. The full AFC protocol in three-level systems can feature both long-lived storage and on-demand recall through collective spin excitations of atoms (spin-wave memory) [20]. However, well-known spin-wave compatible $\mathrm{RE}$ ions offer memory bandwidths of only a few $\mathrm{MHz}$ due to the small frequency spacing between the spin sublevels, thereby hindering the protocol's suitability for a broadband memory [21,22].

Another important avenue for broadband memory is the off-resonant Raman protocol, which features an all-optically controlled spin-wave memory $[23,24]$. As this scheme relies on the "virtual" absorption of light with far-off resonant coupling, it can be used for light storage in atomic media with inhomogeneously broadened lines. Broadband Raman memories have been implemented in warm atomic ensembles which exhibit Doppler broadening [25]. The operation of these memories in the quantum domain, however, has proven difficult due to large four-wave mixing (FWM) noise, which cannot be eliminated using standard filtering techniques [26,27]. Noiseless broadband Raman memories have been achieved in diamond (via storage on phononic transitions) [28] and in ladder-type three-level systems (via storage on optical transitions) [29,30], at the expense of losing the long-lived storage capability that comes with spin storage levels. Lasercooled atoms provide a viable solution for a broadband spinwave Raman memory, as demonstrated with the storage of 

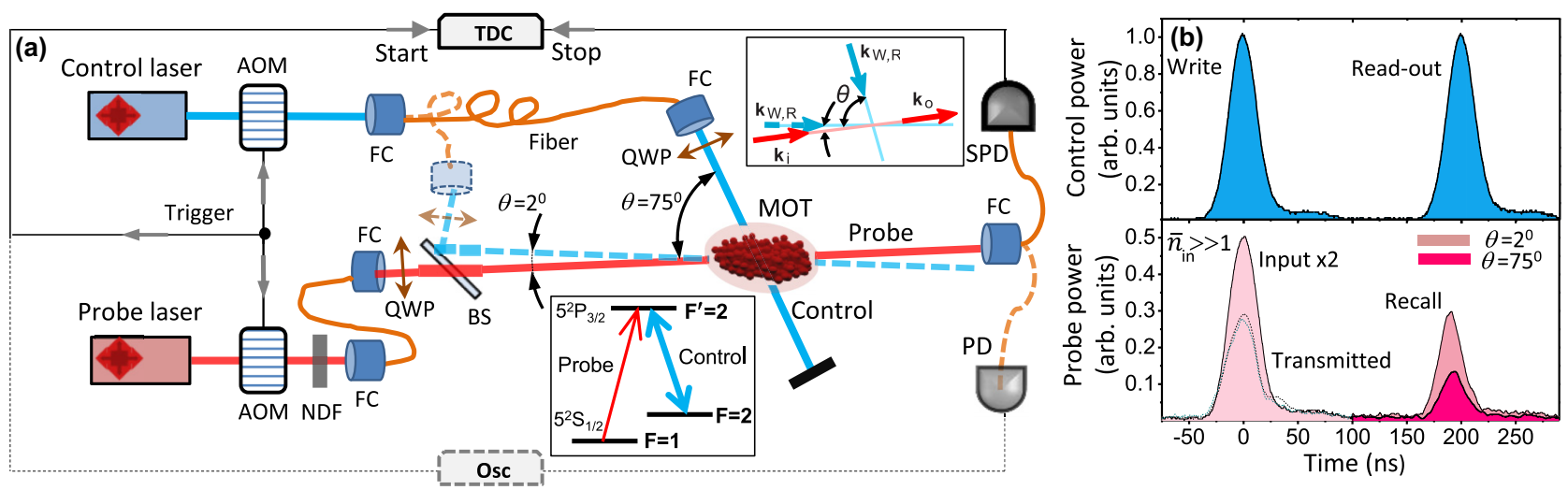

FIG. 1. Experimental setup for implementing ATS memory: (a) Control (blue) and probe (red) beams are derived from two independent continuous-wave lasers and then shaped into short pulses using acousto-optic modulators (AOM). After an adjustable attenuation of the probe beam with neutral density filters (NDF), both beams are coupled into single-mode fibers (FC), and decoupled back to free space on a separate bench where a magneto-optic-trap (MOT) apparatus is located. Following the polarization control using quarter-wave plates (QWP), the beams are overlapped in the atomic cloud (released from MOT) with a separation angle $(\theta)$ of either $2^{\circ}$ via a beam splitter (BS) (dashed traces) or $75^{\circ}$ (solid). After coupling into a fiber, the output probe is detected using either a standard photodiode (PD) for $\bar{n}_{\text {in }} \gg 1$ (in dashed line) or a single-photon detector (SPD) for $\bar{n}_{\text {in }} \leqslant 1$. The arrival times of the detected signals (including a directly transmitted probe) are recorded either on an oscilloscope (Osc) or time-to-digital convertor (TDC), respectively, triggered by a function generator (not displayed). (b) 30-ns-long probe pulses with $\bar{n}_{\text {in }} \gg 1$ are stored and recalled in ATS memory (lower panel) at the $2^{\circ}$ (light) or $75^{\circ}$ (dark) separation angles using write and read-out control pulses (upper panel). Each trace is normalized to its maximum.

7-ns-long nonclassical light pulses [11]. However, the efficient storage of sub-ns pulses (GHz bandwidths) in these systems is technically very demanding in terms of optical depth and coupling-field power, due to the inherent adiabatic operation of the Raman protocol, which exhibits unfavorable bandwidth scaling compared to fast memory protocols $[23,31]$.

The Autler-Townes splitting (ATS) memory protocol [32] combines the inherently fast storage of photon-echo techniques with all-optically controlled spin storage of Ramantype adiabatic memories to overcome these intrinsic and technical limitations. In this Rapid Communication, we experimentally explore the ATS protocol's exceptional robustness to various noise processes and spin decoherence, and demonstrate an ultralow-noise cold-atom memory that is the result of the ATS memory's favorable noise scaling plus the use of all-spatial filtering for the control field. This memory operates optimally much faster than the timescales associated with atomic motion-induced spin decoherence under limited conditions given by the small optical depth and large phase mismatch. By storing short laser pulses with a high signalto-noise ratio for a mean-photon number as small as 0.1, we show that such a memory operation is suitable for quantum information processing [33]. Finally, by exploiting the simple pulse-area-based operation of the ATS protocol, we experimentally realize a temporal beam-splitting process at the single-photon level to demonstrate memory-based pulse manipulation capabilities.

In our experiments, we use a $\Lambda$-type three-level system within the ${ }^{87} \mathrm{Rb}$ atom's electronic structure, comprising two ground hyperfine levels $(|F=1\rangle \equiv|g\rangle$ and $|F=2\rangle \equiv|s\rangle$, energetically spaced by $6.83 \mathrm{GHz})$ and an excited level $\left(\mid F^{\prime}=\right.$ $2\rangle \equiv|e\rangle)$ on the 780-nm $D_{2}$ transition [inset of Fig. 1(a)]. The atoms are laser cooled in a standard magneto-optic trap (as described in Ref. [32]), followed by further subDoppler cooling, and finally optical pumping for populating the atoms in the $|g\rangle$ level. The atomic cloud is probed for light storage/retrieval after releasing it for a 6-ms time of flight, which yields an optical depth of $d \approx 10$.

We implement the ATS protocol in this system for the storage of weak probe laser pulses (resonant with the $|g\rangle \rightarrow|e\rangle$ transition), containing an average number of photons between $\bar{n}_{\text {in }}=0.1$ and $4 \times 10^{6}$. In this protocol [32], a strong control field (coupled to the $|s\rangle \rightarrow|e\rangle$ transition) with the pulse area of $2 \pi$ dynamically splits the homogeneously broadened natural absorption line into two peaks, as per the Autler-Townes effect. Upon absorption of the probe pulses, optical coherence is transiently mapped between the ground $(|g\rangle)$ and excited level $(|e\rangle)$, and then efficiently transferred onto the ground levels $(|g\rangle$ and $|s\rangle)$ as a collective spin excitation for storage [writing stage, Fig. 1(b)]. Retrieval is accomplished after an adjustable time by applying a second control pulse with a pulse area of $2 \pi$, which reconstructs the photonic signal, following a brief reestablishment of coherence between $|g\rangle$ and $|e\rangle$ (read-out stage). Such a nonadiabatic, pulse-area-based operation is similar to photon-echo protocols in inhomogeneously broadened systems $[23,34]$ which require $\pi$ pulses for optimal spin-wave storage and read-out. Both photon-echo and ATS memory efficiencies can approach unity, with a minimal demand on optical depth and control-field power when probe pulses are much shorter than the coherence decay time of the excited level. Compared to the adiabatic memory schemes, such as the off-resonant Raman and electromagnetically induced transparency (EIT) protocols, these relaxed requirements for broadband memory $[32,35]$ provide a great advantage for eliminating control-field-related noise, which is an increasing function of optical depth and/or control intensity $[1,3,27]$. Furthermore, the inherently high-speed operation of ATS memory enables all-spatial filtering of the control noise under certain conditions, which are not accessible to adiabatic memory techniques, as described in the following. 
In our implementation, sketched in Fig. 1(a), the direction of the control beams (represented by wave vectors of $\mathbf{k}_{\mathrm{W}}$ for the write pulse and $\mathbf{k}_{R}$ for the read-out pulse) relative to the input probe beam $\left(\mathbf{k}_{\mathbf{i}}\right)$ determines the direction of the output (retrieved) probe $\left(\mathbf{k}_{\mathrm{o}}=\mathbf{k}_{\mathrm{i}}-\mathbf{k}_{\mathrm{W}}+\mathbf{k}_{\mathrm{R}}\right)$. The relative angles play a key role in both the memory's efficiency and the amount of noise stemming from the control field [36]. In the experiments here, output probe pulses are retrieved in the same direction as the input probe pulses ("forward recall" with $\mathbf{k}_{\mathrm{i}}=\mathbf{k}_{\mathrm{o}}$ ), using copropagating write and read-out control pulses $\left(\mathbf{k}_{\mathrm{W}}=\mathbf{k}_{\mathrm{R}}\right)$ which, despite technical ease, limits the theoretical maximum memory efficiency to $54 \%$ due to reabsorption [32]. In this arrangement, we spatially separate the probe and control beams that overlap in the atomic cloud by introducing an angle $\theta$ between them. While this angle allows for substantial extinction of the control photons from the probe spatial mode before detection, it also leads to a spatial phase grating for the stored spin wave with a period of $\kappa=2 \pi /|\Delta \mathbf{k}|$, where $\Delta \mathbf{k}=\mathbf{k}_{\mathrm{i}}-\mathbf{k}_{\mathrm{W}}$ is imposed by conservation of momentum (phase-matching condition) [36,37].

To begin, as in many previous memory implementations with cold atoms, we set a small separation angle $\theta=2^{\circ}$ [dashed control-beam trace in Fig. 1(a)], resulting in a controlfield extinction of $\approx 40 \mathrm{~dB}$, as well as $\kappa \approx 23 \mu \mathrm{m}$. With this spin-wave periodicity, approximately $100 \mu$ s would have to elapse before our cold atoms (at a temperature of $50 \mu \mathrm{K}$ ) would diffuse and thus "erase" the spin-wave grating during storage, due to motional decoherence. Moreover, for additional spatial filtering of the control field, we collect the probe beam using a single-mode fiber, which increases the isolation up to $65-70 \mathrm{~dB}$.

In this first configuration, we assess the performance of our memory using probe pulses with a large mean-photon number $\left(\bar{n}_{\text {in }} \approx 4 \times 10^{6}\right)$. We store 30 -ns-long Gaussian pulses (at full width at half maximum) and recall after $200 \mathrm{~ns}$, using the write and read-out control pulses with the same temporal profile as the probe, and a peak power of $\approx 20 \mathrm{~mW}$, which gives a pulse area of about $2 \pi$ [Fig. 1(b)]. We measure the memory efficiency $\eta=23 \%$, a threefold improvement upon our first demonstration [32], but still smaller than the theoretical maximum of $38 \%$ for $d=10$ in the forward-recall configuration. This deviation from the theoretical maximum is mainly because of the magnetic-field-induced spin-wave decoherence (as will be further discussed), and partly due to the spatially nonuniform control power that induces additional decoherence during the transient storage between the ground and excited level. Furthermore, we estimate the average number of control photons leaking to the probe mode to be a few hundreds, which is still much smaller than the probepulse photon number. Despite a negligibly small influence in this large mean-photon-number regime, this noise is a major obstacle for single-photon-level memory operation.

While control-field noise may be eliminated by a single optical or atomic filter in narrowband light storage experiments [38], broadband memory implementations typically require the use of several spectral filters for sufficiently large noise isolation, at the expense of additional signal loss and complexity $[11,39]$. Instead of this spectral filtering approach, we implement spatial filtering by increasing the separation angle from $2^{\circ}$ to $75^{\circ}$ [solid control-beam trace in Fig. 1(a)]. To
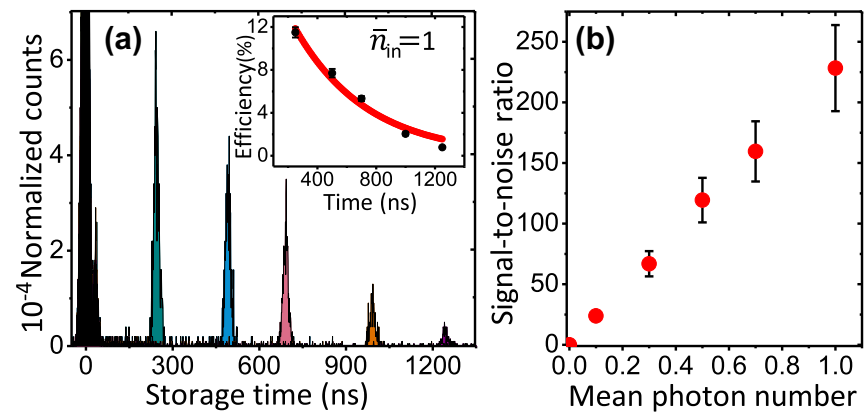

FIG. 2. Single-photon-level ATS memory. (a) Five detection histograms (each normalized to the total number of storage-and-recall attempts $N=5 \times 10^{4}-9 \times 10^{5}$ ) recorded for five different storage times, using 30-ns-long input pulses with $\bar{n}_{\text {in }}=1$. The inset shows the decay of memory efficiency, which is fitted to an exponential curve (solid line). (b) Signal-to-noise ratio (SNR) vs mean-photon number $\left(\bar{n}_{\text {in }}\right)$ for 30-ns-long probe pulses stored in ATS memory for $200 \mathrm{~ns}$. The detection probabilities for the recalled probe ( $p_{\mathrm{s}}$ for a given $\bar{n}_{\text {in }} \neq 0$ ) and noise ( $p_{\mathrm{n}}$ for $\left.\bar{n}_{\text {in }}=0\right)$ are the ratios of the total detection counts (over $\Delta t=50 \mathrm{~ns}$ centered around the recall time) to their respective $N=10^{5}-9 \times 10^{5}$, depending on $\bar{n}_{\text {in }}$.

our detriment, a wide separation angle also induces significant motional decoherence, due to a small-periodicity spin-wave grating $(\kappa \approx 0.65 \mu \mathrm{m})$, which would be washed out over a timescale of only $\approx 1.5 \mu \mathrm{s}$ at a cloud temperature of $50 \mu \mathrm{K}$. While this motional decoherence can be reduced by zeroing the spin-wave vector using two pairs of engineered Raman beams and an auxiliary spin level [40], we instead take advantage of the fast storage and retrieval of the ATS protocol, which enables efficient memory operation at timescales much shorter than the motional decoherence time. This strategy is not feasible for optimal adiabatic memory implementations that require microseconds-long probe pulses in this limitedoptical-depth experimental condition [35].

With this trade-off between the large control-field filtering and limited storage times, we observe that the number of control photons leaking to the probe mode reduces from $\approx 300$ to the single-photon level (over four orders of magnitude extinction), whereas the memory efficiency decreases by only a factor of $\approx 2$ as compared to the small-angle setting for the same storage time [Fig. 1(b)]. We attribute this degradation of memory efficiency at large angles to the limited interaction region that arises from a spatial mismatch between the probe and control beams, having diameters comparable to the size of our atomic cloud.

After substantially isolating the probe beam from stray control photons, we now evaluate the operation of ATS memory at the single-photon level with time-resolved photoncounting measurements using a single-photon detector (SPD) and a time-to-digital converter (TDC). First, we demonstrate the storage and on-demand retrieval of the 30-ns-long probe pulses with $\bar{n}_{\text {in }}=1$, as shown in Fig. 2(a). The storage time is varied between 250 and 1250 ns by changing the time difference between the write and read-out pulses. We observe that memory efficiency drops from $\eta=(11.5 \pm$ $0.5) \%$ to $(0.8 \pm 0.1) \%$ over this time interval, which we attribute to spin-wave decoherence [inset of Fig. 2(a)]. The 
(a)
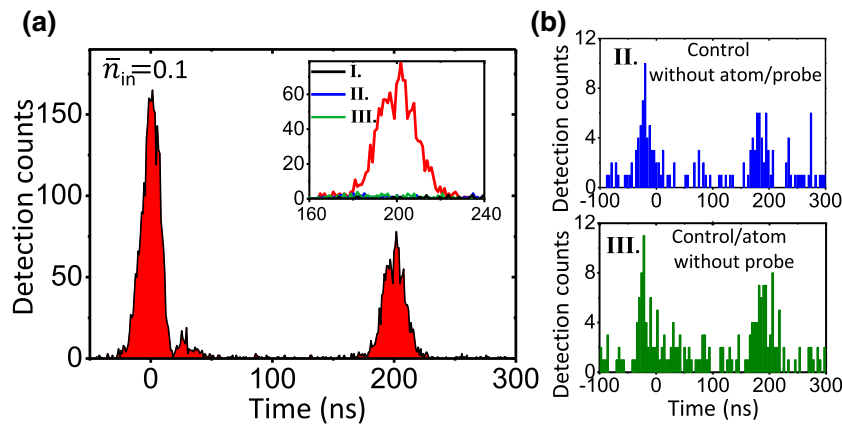

FIG. 3. Noise characterizations: (a) Detection histogram for the storage of 20-ns-long probe pulses for $200 \mathrm{~ns}$ with $\bar{n}_{\text {in }}=0.1$ and $N=1.1 \times 10^{6}$, using $1 \mathrm{~ns} / \mathrm{bin}$. Detections at around zero time are due to the nonabsorbed (transmitted) part of the input probe in the memory medium. The inset shows detections for recalled photons, with respect to noise that is measured in three configurations: (I) Probe without control/atoms, (II) control without probe/atoms, and (III) control/atoms without probe. (b) Detection histograms with larger time bins (4 ns/bin) for the noise measured in (II) (upper panel) and (III) (lower panel).

memory lifetime (at $1 / e$ ) is $490 \pm 60 \mathrm{~ns}$ and is close to the one measured for the small-angle setting $(\approx 650 \mathrm{~ns})$, indicating that spin decoherence due to nonzero ambient magnetic fields dominates over the motional decoherence in our experiments.

Second, we lower the mean-photon number of the input probe pulses below unity and characterize the signal-to-noise ratio $\mathrm{SNR}=\left(p_{\mathrm{s}}-p_{\mathrm{n}}\right) / p_{\mathrm{n}}$ as a function of $\bar{n}_{\text {in }}$ after $200-\mathrm{ns}$ storage, where $p_{\mathrm{s}}$ and $p_{\mathrm{n}}$ are independently measured detection probabilities for retrieved probe and noise (in the absence of probe) after $N$ trials, respectively [Fig. 2(b)]. We observe an almost-linear dependence of SNR on $\bar{n}_{\text {in }}$, and measure a $\mathrm{SNR}=24 \pm 5$ for $\bar{n}_{\text {in }}=0.1$.

Third, we investigate the source and influence of the observed residual noise in a more demanding memory implementation: one with a larger bandwidth and lowest meanphoton number. We decrease the duration of the both probe and control pulses from 30 to $20 \mathrm{~ns}$ (technically the shortest possible in our setup), while increasing the control power to maintain the pulse area near $2 \pi$. We set $\bar{n}_{\text {in }}=0.1$ for probe pulses, and store them in ATS memory for $200 \mathrm{~ns}$ with $\eta=(12.5 \pm 0.4) \%$ [Fig. 3(a)]. After $N=1.1 \times 10^{6}$ attempts, we measure the total number of detection counts (over a time window of $\Delta t=30 \mathrm{~ns}$ centered around the recall) for the stored-and-recalled signal to be $N_{\mathrm{s}}=1357 \pm 40$, and for noise to be $N_{1}=5 \pm 2, N_{2}=22 \pm 5$, and $N_{3}=36 \pm 6$ for three configurations: (I) probe without atom/control, (II) control without atom/probe, and (III) control/atom without probe, respectively [inset of Fig. 3(a)]. Comparing the noise results shows that the main noise component is due to photons from the scattered control field.

We further analyze the detection histograms from configurations (II) and (III) with larger bin sizes [Fig. 3(b)], showing two distinct peaks with a 200-ns separation, corresponding to the time difference between the write and read-out pulses, but shifted backward in time by about 24 ns compared to their original arrival times. We measure nearly the same number of counts for each peak in both configurations
$\left(N_{2}^{\mathrm{W}}=41 \pm 6\right.$ and $N_{3}^{\mathrm{W}}=41 \pm 6$ for writing, and $N_{2}^{\mathrm{R}}=31 \pm 6$ and $N_{3}^{\mathrm{R}}=38 \pm 6$ for reading, respectively), confirming that the stray-control photons are the main source of the residual noise, and there is no measurable four-wave mixing noise.

With these noise contributions [all included in configuration (III)], we determine the unconditional-noise detection probability per pulse (excluding the overall efficiency factor of $\eta_{\mathrm{t}} \approx 0.1$ after memory for the worst-case scenario) to be $p_{\mathrm{n}}=$ $N_{3} / N \times\left(1 / \eta_{\mathrm{t}}\right)=(3.3 \pm 0.5) \times 10^{-4}$, which leads to SNR $=$ $37 \pm 6$ for the stored-and-recalled 20 -ns-long probe pulses. Based on this value, the fidelity of our memory is estimated as $\mathcal{F}=1-1 / \mathrm{SNR}=0.97 \pm 0.01$ (if quantum states were stored), showing the suitability of ATS memory for the highfidelity storage of photonic quantum states.

Although the scattered control field contributes the only measurable noise component in our experiments, it is worth discussing other possible noise sources. In particular, the large angular separation between the probe and control fields violates the phase-matching condition for the FWM noise, rendering it insignificant. Indeed, with a cloud size $L \approx 4 \mathrm{~mm}$, we estimate that FWM effects are not appreciable for $\theta>$ $\theta_{\mathrm{th}}=\sqrt{(\lambda / 2 \pi L)} \approx 2^{\circ}[41]$.

For angles smaller than $\theta_{\text {th }}$ (in all quantum memory protocols), the FWM noise depends strongly on the optical depth (d) and system-specific parameters that are optical decoherence rate between the ground and excited levels $\left(\gamma_{\mathrm{ge}}\right)$ and the energy spacing between the two ground levels $\left(\Delta_{\mathrm{gs}}\right)$. The noise strength is characterized as $\chi=\left(\Omega^{\prime} d \gamma_{\mathrm{ge}}\right) /\left(\Omega \Delta_{\mathrm{gs}}\right)$, where $\Omega^{\prime}$ and $\Omega$ refer to the control Rabi frequency coupling to the probe and storage transitions, respectively $[1,42,43]$. The noise probability scales as $e^{2 \chi}$ and $\chi^{2}$ in the strong $(\chi \gg 1)$ and weak $(\chi \ll 1)$ FWM regimes, respectively. With an optical depth of 10 , as in our current setup, FWM noise $(\chi=0.015 \ll 1)$ would be insignificant using any memory protocol that realizes optimal storage. However, its influence can be drastic as the memory bandwidth increases. In this context, optimal broadband light storage via the ATS protocol offers a significant advantage over adiabatic protocols due to lower optical depth requirements (typically, by a factor of 5-10): For example, an optimal storage of 5-ns pulses using the ATS protocol $(d=100, \chi=0.15)$ would yield a factor of $\approx 40$ less FWM noise than EIT- or Raman-protocol-based memories $(d=600, \chi=0.9)$ [32,35]. In addition, cold and ultracold atoms provide advantages for low-noise memory as compared to their warm counterparts: The elimination of FWM noise is challenging in warm atoms mainly due to Doppler broadening and buffer-gas-induced collisional broadening of $\gamma_{\mathrm{ge}}$, which leads to at least an order of magnitude larger $\chi\left(\propto \gamma_{\text {ge }}\right)$.

Moreover, the reduced demand on control power (typically by a factor of 4-16) makes the ATS memory more robust to various noise processes compared to EIT- and Ramanprotocol memories. While FWM noise scales quadratically with control intensity, other noise contributions such as spontaneous off-resonant Raman scattering and resonant scattering exhibit a nearly linear intensity dependence [26,27]. Consequently, in combination with its inherent advantage from the optical depth dependence of noise sources, cold-atom broadband ATS memory can exhibit an orders-of-magnitude lower 


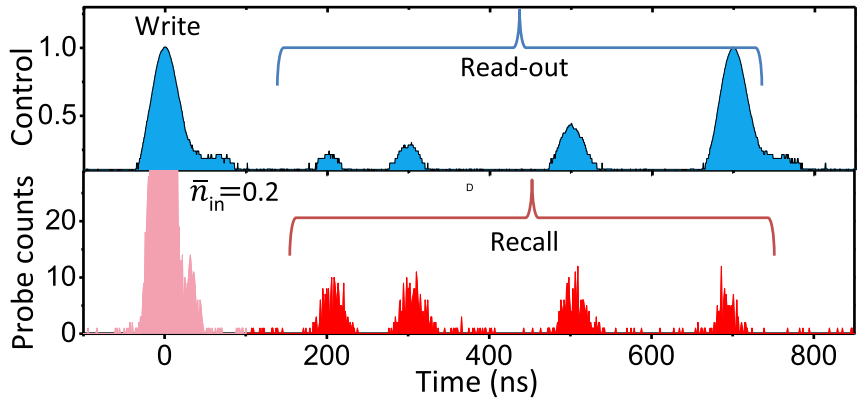

FIG. 4. Temporal beam splitting at the single-photon level. Upper panel: A write control pulse and multiple read-out pulses, each of 30-ns duration, are used for the retrieval of a single stored mode at different times. Control pulses, shown in units proportional to electric field/Rabi frequency, have pulse areas $\leqslant 2 \pi$ depending on the fraction of spin coherence desired upon retrieval. These pulses are normalized to the height of the $2 \pi$ write pulse. Lower panel: Detection histogram obtained after $N=4 \times 10^{5}$ attempts for the storage and retrieval of a 30 -ns-long probe pulse with $\bar{n}_{\text {in }}=0.2$, using the control-pulse sequence in the upper panel.

noise probability as compared to state-of-the-art broadband spin-wave memories.

Finally, we demonstrate temporal beam splitting using ATS memory at the single-photon level (Fig. 4) [32]. We store 30-ns-probe pulses with $\bar{n}_{\text {in }}=0.2$ using a write pulse with a pulse area of $2 \pi$, as in the standard ATS scheme. In contrast, the retrieval is sequentially realized at different times using multiple read-out control pulses, each with a pulse area smaller than $2 \pi$ (except the last one that retrieves all the remaining spin coherence). This process results in an output photon in a superposition of multiple temporal bins (lower panel in Fig. 4), demonstrating the temporal beam-splitting capability of single-photon-level ATS memory.

Beyond these proof-of-principle demonstrations, future implementations of ATS memory could benefit from the use of ultracold atoms in an optical-dipole trap [44]. First of all, the storage time can be extended to the millisecond regime by using clock spin states [45], or even to the seconds timescale [46], given the long hold times of atoms in optical-dipole traps. Particularly, a Bose-Einstein condensate (BEC) offers both long storage times and flexibility of all-spatial filtering simultaneously, as atomic diffusion in a BEC is virtually absent [47]. Second, the memory efficiency can reach near unity in the backward-recall configuration at larger optical depths $(d \geqslant 100)$, benefiting the inherently large densities of ultracold quantum gases [48]. Third, the memory bandwidth can be extended from $20 \mathrm{MHz}$ to the $\mathrm{GHz}$ regime, by forming the $\Lambda$ system in the $D_{1}$ manifold of $\mathrm{Rb}$, which provides near$\mathrm{GHz}$ excited-level spacing, and by using fast electro-optic components for tailoring control pulses. With these moderate improvements, the ATS approach offers high-performance quantum memories for quantum networks.

In conclusion, we experimentally implemented the ATS protocol in a cold $\mathrm{Rb}$ gas for the storage of single-photon-level optical pulses as collective spin excitations. Ultralow-noise memory operation is achieved by eliminating the contamination of the control-field photons with all-spatial filtering techniques, at the expense of limited storage times. While the residual noise is dominated by the stray control field, the four-wave mixing noise is not a limiting effect in our experimental configuration. These results indicate that coldatom-based ATS memory is a preeminent option for quantum light storage.

We thank Dr. Khabat Heshami for useful discussions, and Dr. Alex Lvovsky for lending us a single-photon detector for our initial measurements. We appreciate generous technical support from Paul Davis and Greg Popowich. We gratefully acknowledge funding from the Natural Science and Engineering Research Council of Canada (NSERC RGPIN-201406618), Canada Foundation for Innovation (CFI), Canada Research Chairs Program (CRC), Canadian Institute for Advanced Research (CIFAR), Alberta Innovates (AITF), and the University of Alberta.
[1] N. Lauk, C. O'Brien, and M. Fleischhauer, Phys. Rev. A 88, 013823 (2013).

[2] P. J. Bustard, D. G. England, K. Heshami, C. Kupchak, and B. J. Sussman, Opt. Lett. 41, 5055 (2016).

[3] L. Ma, O. Slattery, and X. Tang, Mod. Phys. Lett. B 32, 1830001 (2018).

[4] J. Nunn, K. Reim, K. C. Lee, V. O. Lorenz, B. J. Sussman, I. A. Walmsley, and D. Jaksch, Phys. Rev. Lett. 101, 260502 (2008).

[5] W. Tittel, M. Afzelius, T. Chaneliére, R. Cone, S. Kröll, S. Moiseev, and M. Sellars, Laser Photonics Rev. 4, 244 (2009).

[6] N. Sangouard, C. Simon, H. de Riedmatten, and N. Gisin, Rev. Mod. Phys. 83, 33 (2011).

[7] S. A. Moiseev and S. Kröll, Phys Rev. Lett. 87, 173601 (2001).

[8] B. Kraus, W. Tittel, N. Gisin, M. Nilsson, S. Kröll, and J. I. Cirac, Phys. Rev. A 73, 020302 (2006).

[9] A. L. Alexander, J. J. Longdell, M. J. Sellars, and N. B. Manson, Phys. Rev. Lett. 96, 043602 (2006).
[10] G. Hétet, M. Hosseini, B. M. Sparkes, D. Oblak, P. K. Lam, and B. C. Buchler, Opt. Lett. 33, 2323 (2008).

[11] D.-S. Ding, W. Zhang, Z.-Y. Zhou, S. Shi, B.-S. Shi, and G.-C. Guo, Nat. Photonics 9, 332 (2015).

[12] M. Mazelanik, M. Parniak, A. Leszczyński, M. Lipka, and W. Wasilewski, npj Quantum Inf. 5, 22 (2019).

[13] M. Afzelius, C. Simon, H. de Riedmatten, and N. Gisin, Phys. Rev. A 79, 052329 (2009).

[14] E. Saglamyurek, N. Sinclair, J. Jin, J. A. Slater, D. Oblak, F. Bussières, M. George, R. Ricken, W. Sohler, and W. Tittel, Nature (London) 469, 512 (2011).

[15] C. Clausen, I. Usmani, F. Bussiéres, N. Sangouard, M. Afzelius, H. de Riedmatten, and N. Gisin, Nature (London) 469, 508 (2011).

[16] E. Saglamyurek, J. Jin, V. B. Verma, M. D. Shaw, F. Marsili, S. W. Nam, D. Oblak, and W. Tittel, Nat. Photonics 9, 83 (2015). 
[17] A. Tiranov et al., Optica 2, 279 (2015).

[18] N. Sinclair et al., Phys. Rev. Lett. 113, 053603 (2014).

[19] E. Saglamyurek, N. Sinclair, J. A. Slater, K. Heshami, D. Oblak, and W. Tittel, New J. Phys. 16, 065019 (2014).

[20] M. Afzelius et al., Phys. Rev. Lett. 104, 040503 (2010).

[21] N. Timoney, I. Usmani, P. Jobez, M. Afzelius, and N. Gisin, Phys. Rev. A 88, 022324 (2013).

[22] M. Gündogan, P. M. Ledingham, K. Kutluer, M. Mazzera, and H. de Riedmatten, Phys. Rev. Lett. 114, 230501 (2015).

[23] A. V. Gorshkov, A. André, M. D. Lukin, and A. S. Sørensen, Phys. Rev. A 76, 033805 (2007).

[24] J. Nunn, I. A. Walmsley, M. G. Raymer, K. Surmacz, F. C. Waldermann, Z. Wang, and D. Jaksch, Phys. Rev. A 75, 011401(R) (2007).

[25] K. F. Reim, J. Nunn, V. O. Lorenz, B. J. Sussman, K. C. Lee, N. K. Langford, D. Jaksch, and I. A. Walmsley, Nat. Photonics 4, 218 (2010).

[26] P. S. Michelberger, Ph.D. thesis, University of Oxford, 2015.

[27] J. Nunn et al., Phys. Rev. A 96, 012338 (2017).

[28] D. G. England, K. A. G. Fisher, J.-P. W. MacLean, P. J. Bustard, R. Lausten, K. J. Resch, and B. J. Sussman, Phys. Rev. Lett. 114, 053602 (2015).

[29] K. T. Kaczmarek et al., Phys. Rev. A 97, 042316 (2018).

[30] R. Finkelstein, E. Poem, O. Michel, O. Lahad, and O. Firstenberg, Sci. Adv. 4, eaap8598 (2018).

[31] A. V. Gorshkov, A. André, M. Fleischhauer, A. S. Sørensen, and M. D. Lukin, Phys. Rev. Lett. 98, 123601 (2007).

[32] E. Saglamyurek, T. Hrushevskyi, A. Rastogi, K. Heshami, and L. J. LeBlanc, Nat. Photonics 12, 774 (2018).

[33] X. Ma, B. Qi, Y. Zhao, and H.-K. Lo, Phys. Rev. A 72, 012326 (2005).

[34] A. V. Gorshkov, A. Andre, M. D. Lukin, and A. S. Sorensen, Phys. Rev. A 76, 033806 (2007).
[35] A. Rastogi, E. Saglamyurek, T. Hrushevskyi, S. Hubele, and L. J. LeBlanc, Phys. Rev. A 100, 012314 (2019).

[36] K. Surmacz, J. Nunn, K. Reim, K. C. Lee, V. O. Lorenz, B. Sussman, I. A. Walmsley, and D. Jaksch, Phys. Rev. A 78, 033806 (2008).

[37] B. Zhao, Y.-A. Chen, X.-H. Bao, T. Strassel, C.-S. Chuu, X.-M. Jin, J. Schmiedmayer, Z.-S. Yuan, S. Chen, and J.-W. Pan, Nat. Phys. 5, 95 (2008).

[38] D. T. Stack, P. J. Lee, and Q. Quraishi, Opt. Express 23, 6822 (2015).

[39] S. E. Thomas, T. M. Hird, J. H. D. Munns, B. Brecht, D. J. Saunders, J. Nunn, I. A. Walmsley, and P. M. Ledingham, arXiv:1905.00042.

[40] Y. Jiang, J. Rui, X.-H. Bao, and J.-W. Pan, Phys. Rev. A 93, 063819 (2016).

[41] A. J. MacRae, Ph.D. thesis, University of Calgary, 2012.

[42] J. Geng, G. T. Campbell, J. Bernu, D. B. Higginbottom, B. M. Sparkes, S. M. Assad, W. P. Zhang, N. P. Robins, P. K. Lam, and B. C. Buchler, New J. Phys. 16, 113053 (2014).

[43] G. Romanov, C. O’Brien, and I. Novikova, J. Mod. Opt. 63, 2048 (2016).

[44] C.-S. Chuu, T. Strassel, B. Zhao, M. Koch, Y.-A. Chen, S. Chen, Z.-S. Yuan, J. Schmiedmayer, and J.-W. Pan, Phys. Rev. Lett. 101, 120501 (2008).

[45] R. Zhao, Y. O. Dudin, S. D. Jenkins, C. J. Campbell, D. N. Matsukevich, T. A. B. Kennedy, and A. Kuzmich, Nat. Phys. 5, 100 (2008).

[46] Y. O. Dudin, L. Li, and A. Kuzmich, Phys. Rev. A 87, 031801(R) (2013).

[47] S. Riedl, M. Lettner, C. Vo, S. Baur, G. Rempe, and S. Dürr, Phys. Rev. A 85, 022318 (2012).

[48] P. Vernaz-Gris, A. D. Tranter, J. L. Everett, A. C. Leung, K. V. Paul, G. T. Campbell, P. K. Lam, and B. C. Buchler, Opt. Express 26, 12424 (2018). 